\title{
Impacto da Política de Reserva de Vagas nas taxas de Evasão na Unesp
}

\author{
Impact of the vacancy reservation policy on Unesp dropout rate
}

\author{
Gladis Massini-Cagliari ${ }^{1}$ \\ Ney Lemke ${ }^{2}$ \\ Iraíde Marques de Freitas Barreiro ${ }^{3}$ \\ Maria Aparecida Custódio Domingues ${ }^{4}$ \\ Fernando Ferrari Putti ${ }^{5}$ \\ Silvana Aparecida Borsetti Gregorio Vidotti ${ }^{6}$
}

Resumo: A evasão é um tema de interesse mundial na área da educação, contudo o entendimento atual sobre o fenômeno é ainda insuficiente, sobretudo quando o objetivo é avaliar políticas efetivas de evasão. Neste artigo o percentual anual de evasão da Unesp é avaliado com base na adoção da política de reserva de vagas. Existem três possibilidades de ingresso - sistema universal (SU), quota por ter estudado em escola pública (EP) e quota por ser PPI (pretos, pardos, indígenas). Os dados foram segmentados por curso e ano de ingresso. A Unesp tem campus em 24 cidades, que reúnem 34 unidades e abrigam 136 diferentes cursos de Graduação. O ingresso para estes cursos é realizado por meio de vestibular com 186 diferentes opções em 63 carreiras. Uma comparação das taxas de evasão permite observar que elas podem depender do modo de ingresso, mas isso ocorre de forma variada ao longo do tempo e depende da área do curso, não sendo significativo. Verificou-se um decréscimo consistente da taxa de evasão global e por categoria de ingresso.

Palavras-chave: Educação superior. Evasão. Reserva de vagas. 
Abstract: Student dropout is a topic of worldwide interest in the field of education research; however, current understanding of the phenomenon remains insufficient, particularly when the objective is to evaluate effective retention policies. The annual dropout rate at UNESP was evaluated based on the admission selection policy. There are three admission categories: the universal exam system; a selection quota for students who studied in public schools; and a selection quota for students who are Black, Brown or Indigenous. The data was classified by course and admittance year. UNESP has campuses in 24 towns, divided into 34 campuses that provide 136 different undergraduate courses. Admission to these courses is conducted by an entrance exam with 186 different options in 63 careers. A comparison of dropout rates shows that these may depend on the admission category, but it varies over time and depends on the course subject. The data also reveal a consistent decrease in the annual dropout rates, according to the admission category.

Keywords: Higher education. School dropout. Vacancy reserve policy.

${ }^{1}$ Universidade Estadual Paulista "Júlio de Mesquita Filho"| Faculdade de Ciências e Letras | Araraquara | SP | Brasil. Contato: gladis.massini-cagliari@unesp.br. ORCID: https://orcid.org/0000-0002-4050-7645

${ }^{2}$ Universidade Estadual Paulista "Júlio de Mesquita Filho" | Instituto de Biociências | Botucatu | SP | Brasil. Contato: ney.lemke@unesp.br. ORCID: https://orcid.org/0000-0001-7463-4303

${ }^{3}$ Universidade Estadual Paulista "Júlio de Mesquita Filho" | Faculdade de Ciências e Letras | Assis | SP | Brasil. Contato: iraide.barreiro@unesp.br. ORCID: https://orcid.org/0000-0001-9203-0351

${ }^{4}$ Universidade Estadual Paulista "Júlio de Mesquita Filho" | Faculdade de Medicina de Botucatu | Botucatu | SP | Brasil. Contato: maria.domingues@ unesp.br. ORCID: https://orcid.org/0000-0002-4023-3410

${ }^{5}$ Universidade Estadual Paulista "Júlio de Mesquita Filho" | Faculdade de Ciências e Engenharias | Tupã | SP | Brasil. Contato: fernando.putti@unesp.br. ORCID: http://orcid.org/0000-0002-0555-9271

${ }^{6}$ Universidade Estadual Paulista "Júlio de Mesquita Filho" | Faculdade de Filosofia e Ciências | Marília |SP | Brasil. Contato: silvana.vidotti@unesp.br. ORCID: https://orcid.org/0000-0002-4216-0374

- Recebido em: 27 de fevereiro 2020

- Aprovado em: 20 outubro 2020

DOI: http://dx.doi.org/10.1590/S1414-40772021000100011

Este é um artigo publicado em acesso aberto sob uma licença Creative Commons https://creativecommons.org/licenses/by-nc/4.0/ 


\section{Introdução}

A evasão é um tema de interesse mundial na área da educação, seja porque impacta economicamente as universidades e a sociedade, seja pelas consequências que causa na vida dos estudantes. Ainda que extensivamente investigada e que exista ampla literatura sobre o tema (ARULAMPALAM; NAYLOR; SMITH, 2001; BLANCHFIELD, 1972; BOOTH; SATCHELL, 1995; BRADLEY; LENTON, 2005; DESJARDINS et al., 1999; ECKSTEIN; WOLPIN, 1999; EHRENBERG; MAVROS, 1995; MALLETE; CABRERA, 1991; PANTAGES; CREEDON, 1978; SIEGFRIED; STOCK, 2001; SIMPSON; BAKER; MELLINGER, 1980; TINTO, 1987), o entendimento sobre o fenômeno é ainda insuficiente, especialmente quando o objetivo é avaliar políticas efetivas de retenção. Um dos poucos consensos que emergem desses estudos é que a evasão é um fenômeno social e que políticas eficientes são aquelas que produzem engajamento entre todos os membros da comunidade universitária (TINTO, 1987).

O uso do termo evasão "está associado a diferentes perspectivas, temporalidades, granularidades e fórmulas", havendo "diferentes concepções e usos do termo em produções acadêmicas" (VITELLI; FRITSCH, 2016, p. 908). Apesar de o INEP disponibilizar em seu site índices de evasão para todos os cursos de graduação do país, com base nos dados colhidos no Censo da Educação, a definição do que se considera evasão e a determinação dos índices para seus cálculos estão associadas à autonomia das instituições de ensino no processo de construção de seus próprios indicadores de gestão (VITELLI; FRITSCH, 2016). Por esta razão, Silva Filho e Lobo (2012, p. 4) consideram que "não há fórmula ideal, porque o cálculo da evasão depende dos critérios e das metodologias adotadas".

A Comissão Especial de Estudos sobre a Evasão nas Universidades Públicas Brasileiras - ANDIFES/ABRUEM/SESu/MEC (1996, p. 28) definiu a evasão dos cursos de graduação como "a saída definitiva do aluno de seu curso de origem, sem concluí-lo". O estudo empreendido pela Comissão indicada pelo MEC estabeleceu que o melhor entendimento da evasão no ensino superior deve considerar uma geração completa. "Por geração completa entende-se aquela em que o número de diplomados ( $\mathrm{ND} /)$, mais o número de evadidos $(\mathrm{Ne})$, mais o número de retidos (Nr) é igual ao número de ingressantes no ano-base (Ni), considerando o tempo máximo de integralização do curso, seja $\mathrm{Ni}=\mathrm{Nd}+\mathrm{Ne}+\mathrm{Nr}$ " (COMISSÃO ESPECIAL DE ESTUDOS SOBRE A EVASÃO NAS UNIVERSIDADES PÚBLICAS BRASILEIRAS, 1996, p. 28). Entretanto, a própria Comissão apresenta algumas deficiências do cálculo adotado. A primeira está relacionada com o fato de o modelo considerar como evadido todo e qualquer 
aluno que, não estando mais vinculado ao curso, não o tenha concluído no prazo máximo de integralização curricular, embora possa ter se transferido ou ingressado em outro curso da própria universidade, por meio de novo vestibular, ou ter reingressado no mesmo curso da universidade, por novo vestibular, com o objetivo de "limpar" seu histórico escolar. Desse modo, tem-se a evasão do curso (quando o aluno deixa o curso, mas pode continuar ou não em outro curso da mesma ou de outra universidade ou não), a evasão da universidade (quando o aluno deixa o curso para ingressar em um curso de graduação em outra universidade) e a evasão do sistema universitário (quando o aluno deixa o curso e não se matricula em outro curso da universidade ou fora dela). Neste artigo, adota-se, como foco, a evasão do curso, uma vez que o interesse é acompanhar turmas de ingressantes em uma mesma categoria de ingresso pelo vestibular; entretanto, a Unesp também monitora os índices de evasão da universidade.

No caso brasileiro, a evasão também tem sido alvo de estudos e de debates, tanto no contexto das universidades públicas como no setor privado. No caso das universidades públicas, há crescente pressão social para que exista um engajamento dessas instituições na redução das desigualdades sociais e raciais no Brasil. A política de cotas é a manifestação mais explícita dessa demanda, apesar de haver poucos estudos sistemáticos que as analisam. Um dos poucos trabalhos que apresenta resultados empíricos é o de Bezerra (2011), que investigou o tema no contexto da UERJ e concluiu que a evasão era menor entre os cotistas.

Schwartzman (2008) apresenta uma perspectiva particularmente crítica sobre o impacto da política de cotas e seu sucesso no contexto brasileiro. Entre as críticas que apresenta, podese citar o baixo impacto, os riscos à qualidade dos profissionais formados por esses sistemas e, finalmente, que a ideia de que as cotas, apesar de bem intencionadas, enfatizam demais aspectos raciais, que não são a origem principal das desigualdades no Brasil.

Constata-se, portanto, a confluência de muitos temas interagentes ao se propor investigar a questão da eficiência da política de cotas em uma Universidade como a Unesp, que possui várias características que a tornam ímpar, como sua extensão geográfica, sua capilaridade, pluralidade e redundância de cursos e sua história.

O principal objetivo deste artigo é discutir e analisar o impacto da política de reserva de vagas nas taxas de evasão, por categoria de ingresso (vestibular universal, versus escola pública e, dentre esses, o grupo dos PPI), por curso. Pretende-se observar se a categoria de ingresso está relacionada com o risco de evasão, nos cursos de graduação na Unesp. Os dados que alimentam a pesquisa são oriundos das bases de dados institucionais. Inicialmente aborda-se o contexto institucional; na sequência são apresentados os resultados obtidos e, finalmente, as conclusões. 


\section{Contexto institucional}

A Unesp foi a primeira das três universidades públicas estaduais paulistas a adotar, em 2013 (para ingresso em 2014), uma política de reserva de vagas para estudantes que realizaram sua formação integralmente em escolas públicas e estabelecendo cotas para estudantes pretos, pardos e índios (PPI), correspondente ao percentual de participação dessa parcela na população geral do estado. Pela primeira vez, naquele vestibular, foi adotado o Sistema de Reserva de Vagas da Educação Básica Pública (SRVEBP).

A Unesp é a universidade estadual mais bem distribuída pelo interior do estado de São Paulo, com câmpus em 24 cidades, que reúnem 34 unidades e abrigam 136 diferentes cursos de Graduação. O ingresso para estes cursos é realizado a partir de concursos vestibulares com 186 diferentes opções em 63 carreiras. São 39 cursos na área de Ciências Biológicas, 54 de Ciências Exatas e 43 na área de Humanidades, distribuídos em 119 Bacharelados, 31 Licenciaturas e 36 Bacharelados/Licenciaturas.

O projeto de inclusão aprovado pela Unesp prevê que 50\% das vagas, para cada curso e turno, sejam preenchidas por estudantes que cursaram integralmente o ensino médio em escolas públicas e que, desse total, 35\% das vagas devem ser reservadas para PPIs (VASCONCELOS; GALHARDO, 2016). A metodologia adotada para a inclusão é o processo classificatório do vestibular, com aproveitamento dos candidatos até o limite de vagas fixadas para cada curso e turno.

De modo progressivo, no decorrer de cinco anos, em 2014, houve reserva de $15 \%$ das vagas para oriundos de escola pública, incluindo os PPIs; a partir daí, reservou-se $25 \%$, em 2015; 35\%, em 2016; 45\%, em 2017; e a meta de 50\% de vagas reservadas para o SRVEBP, das quais $35 \%$ para PPI foi atingida em 2018. Ao completar o período de implantação do SRVEBP, é preciso acompanhar o desempenho dos diferentes grupos de ingressantes pelo vestibular entre as categorias (vestibular universal versus escola pública e, dentre esses, os PPI), a fim de verificar a eficácia do sistema adotado e os seus reflexos, na permanência dos estudantes na universidade.

A evasão é um indicador da qualidade e analisar sua evolução temporal nas três categorias de ingresso permite investigar e quantificar o impacto dessas políticas.

\section{Metodologia: índices anuais de evasão}

Pretende-se analisar e comparar os índices anuais de evasão da universidade em cada categoria de ingresso e por curso, de modo a observar se a categoria de ingresso (SU, EP e PPIs) 
está relacionada com o risco de evasão. Para tal, serão adotadas duas abordagens: análise do percentual médio de evasão de cada turma, acompanhada de um teste pareado indicativo do risco de evasão em cada categoria.

Massini-Cagliari et al. (2018) mostraram que, com o avanço da implementação do sistema de reserva de vagas na Unesp e a sua relação com a permanência nos cursos de graduação da universidade, há necessidade da adoção de um índice anual para acompanhamento da evolução das taxas de evasão. Logo, os autores apresentaram a metodologia adotada para o cálculo dos índices anuais de desistência, nos cursos de Graduação da Unesp e a adoção de um sistema de coleta dos dados da evasão. Serão apresentadas brevemente as razões já apontadas em Massini-Cagliari et al. (2018) para a escolha de um índice anual como ferramenta de acompanhamento, bem como a metodologia de cálculo, já referida nesse trabalho anterior.

Quando se tem como objetivo a observação da eficácia de um sistema de ingresso que apenas acaba de completar seu ciclo de implantação, há que se adotar uma metodologia de coleta e análise dos dados da evasão que seja dinâmico e flexível o bastante para possibilitar o acompanhamento dos dados advindos de grupos cuja dimensão vai se alterando com o avançar dos anos. Tem-se observado que a categoria Sistema Universal vai diminuindo de tamanho a cada ano da implantação do SRVEBP, ao passo que se amplia o percentual reservado aos oriundos de escola pública, e, consequentemente, de PPI. Logo, considerar as taxas de evasão com base na geração completa ou turma cheia pode ser problemático, uma vez que o índice se baseia na observação do comportamento de turmas completas, desde o seu ingresso até que o último aluno seja desligado do curso/da universidade (por qualquer razão: titulação, desistência, transferência interna ou externa, falecimento, entre outras).

Assim sendo, optou-se por considerar como foco, no presente artigo, o percentual anual de desistência em cada turma de ingresso. Para determinar se a evasão é maior ou menor nos diferentes cursos da universidade, foi aplicado um teste pareado comparando as formas de ingresso duas a duas, considerando os cursos como elementos básicos. Com isso, pode-se afirmar se existe ou não diferenças estatisticamente significativas nos percentuais de evasão entre as formas de ingresso.

Conforme já apresentado, Massini-Cagliari et al. (2018) consideram a diferenciação entre ingressantes e veteranos, de modo a, como opção de gestão, focalizar as dificuldades enfrentadas por quem chega ao curso e à universidade pela primeira vez, em confronto com as dificuldades de quem já está há mais tempo no curso. As fórmulas também consideram a diferença entre evasão da universidade ( $\left.\mathrm{E}_{\text {unesp }}\right)$ e do curso ( $\left.\mathrm{E}_{\text {curso }}\right)$.

1) Percentual de evasão nos ingressantes no curso: 


$$
E_{i}=\left\{1-\frac{N_{i}-N_{d}-N t_{e x}-N t_{i n}}{N_{i}}\right\} \times 100
$$

2) Percentual de evasão nos demais matriculados no curso (veteranos):

$$
E_{m}=\left\{\frac{N_{m}-N_{d}-N_{c}-N t_{e x}-N t_{i n}}{N_{m}}\right\} \times 100
$$

3) Percentual de evasão total no curso:

$$
E_{\text {curso }}=E_{i}+E_{m}
$$

4) Percentual de evasão na universidade é calculado pelas mesmas fórmulas, mas considerando todos os inscritos na universidade, onde:

$N_{i}=$ número de ingressantes

$N_{m}=$ número de matriculados

$N_{d}=$ desistência do curso feita pelo aluno

$N_{c}=$ cancelamento feito pela UNESP

$N t_{e x}=$ transferência externa

$N t_{i n}=$ transferência interna

No presente artigo, considera-se como base para a comparação entre as categorias de ingresso no vestibular a fórmula apresentada em (3), que se refere a todos os estudantes que desistiram do curso no decorrer do ano focalizado, independentemente de terem se transferido para outro curso do mesmo ou de outro campus da Universidade.

Baseado na metodologia de cálculo proposta, foi desenvolvido um ambiente de cálculo dos índices anuais de evasão dentro do Sistema da Graduação da Unesp (SISGrad), por meio dos quais é possível extrair informações relativas à evolução dos índices anuais de evasão de cada turma de cada curso de Graduação da Unesp, considerando as categorias de ingresso adotadas no vestibular. A análise focalizará o período de implantação da adoção do SRVEBP no vestibular de 2014 a 2018.

Todas as análises foram realizadas na linguagem Python e o módulo estatístico scikitlearn v. 21 (acessível em https://scikit-learn.org). O código foi executado utilizando o software Jupyter. As diferenças entre os grupos na ocorrência da taxa de evasão foram comparadas pelo teste exato de Fisher (FISHER, 1922). Uma diferença foi considerada estatisticamente significativa se a probabilidade bilateral do resultado observado sob a hipótese nula fosse $\leq 0,05$. As comparações entre as médias das taxas de evasão dos diferentes grupos foram realizadas 
pelo teste $\mathrm{t}$ de Student pareado (STUDENT, 1987). Uma diferença foi considerada estatisticamente significativa se a probabilidade do resultado observado sob a hipótese nula fosse $\leq 0,05$.

\section{Evasão e reserva de vagas}

Como mostra o Gráfico 1, de 2014 a 2018, o índice anual de evasão da Unesp, ou seja, o percentual de alunos que saem da universidade por razões diversas, variou de $6.7 \%$ (em 2016) a 5.1\% (2018). O índice de evasão dos cursos, entretanto, é ligeiramente maior, nesse período, uma vez que, nesse cálculo, também são considerados como tendo desistido do curso (mas não da Unesp ou do sistema superior) os alunos que se transferem para outro curso do mesmo ou de outro campus da universidade.

Gráfico 1 - Índices anuais de evasão dos cursos e da Unesp (2014-2018)

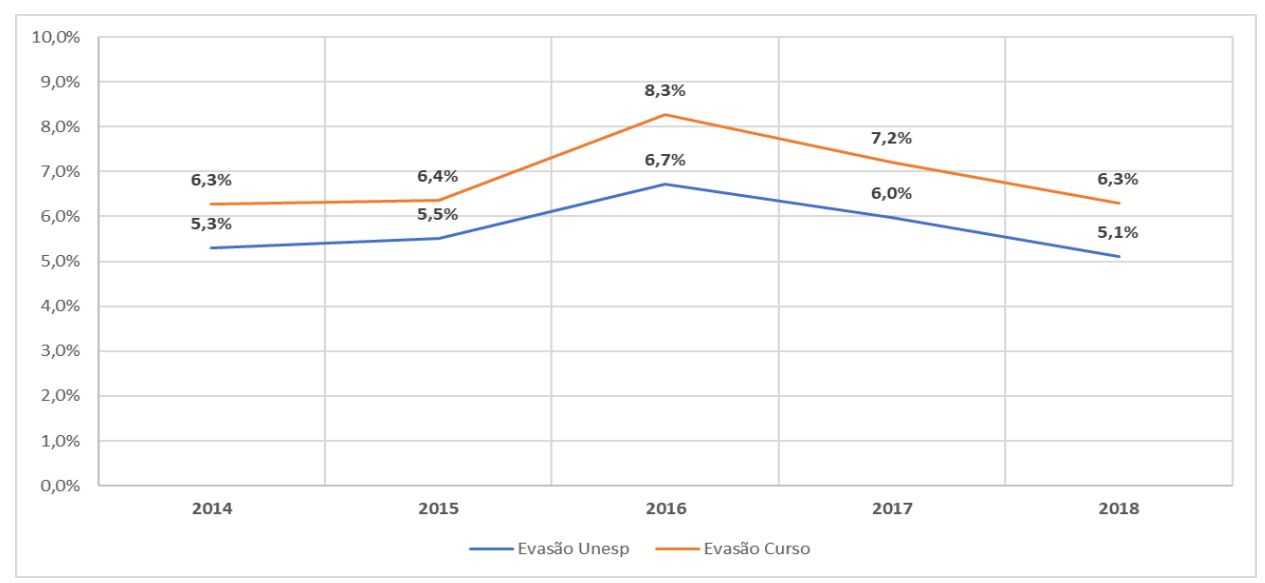

Fonte: Unesp, Sistema de Base de Dados da Graduação - SISGrad: 2014-2018.

Os Gráficos 2 e 3 expressam, respectivamente, em números absolutos, a quantidade de alunos matriculados e evadidos, divididos por categoria de ingresso: SU e EP, correspondente aos candidatos oriundos da escola pública que se inscreveram para disputar as vagas reservadas para o SRVEBP, mas que não se caracterizam para disputar as vagas de PPI, subdivisão do SRVEBP, correspondente a cotas raciais. Observa-se que há uma evolução da variação da dimensão de cada categoria, à medida que vai sendo implantado o SRVEBP no vestibular da Unesp, para ingresso nos cursos de Graduação. Conforme o percentual de vagas reservadas vai crescendo de $15 \%$ (2014) para 25\% (2015), 35\% (2016), 45\% (2017), até atingir a meta de 50\% em 2018, a dimensão relativa dos ingressantes pelo sistema universal vai diminuindo e a proporção de EP e de PPI vai gradativamente aumentando. É importante ressaltar, entretanto, que, apesar de a meta de 50\% de vagas reservadas à escola pública ter sido atingida em 2018 , 
a distribuição desses percentuais pela população de alunos da Unesp ainda não se estabilizou em metade para cada categoria (SU e SRVEBP), uma vez que esse equilíbrio só será atingido quando todos os alunos ingressantes no período de implantação progressiva do sistema de inclusão ou anteriormente a ele já tiverem completado seus cursos.

Gráfico 2 - Total de alunos matriculados, por categoria de ingresso (SU, EP, PPI)

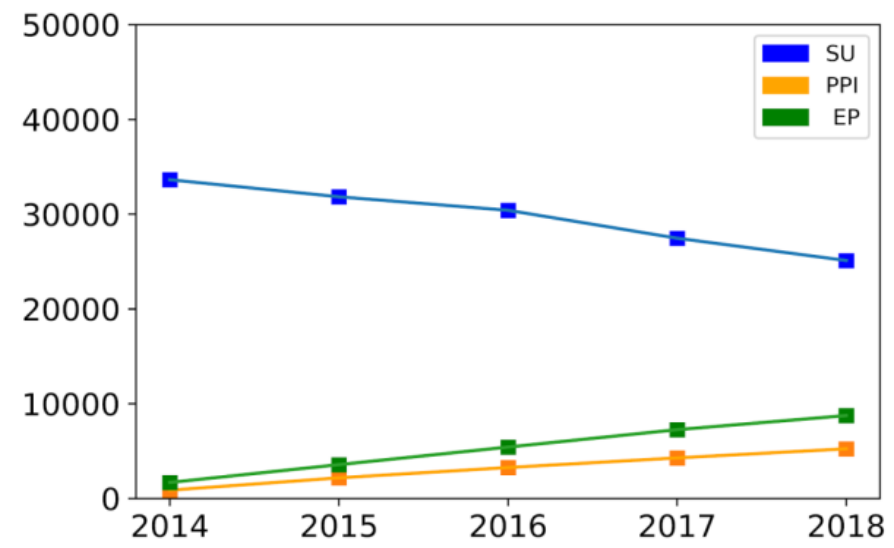

Fonte: Unesp, Sistema de Base de Dados da Graduação - SISGrad : 2014-2018.

\section{Gráfico 3 - Total de alunos evadidos, por categoria de ingresso (SU, EP, PPI)}

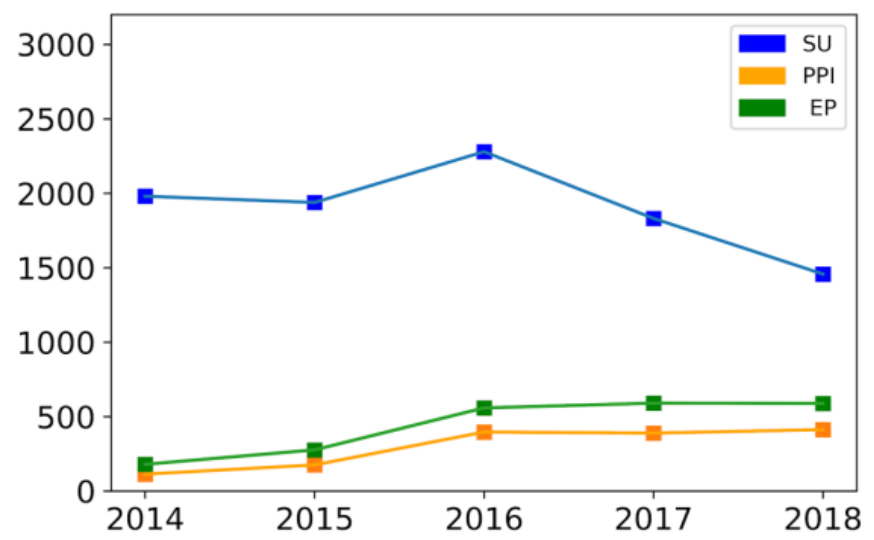

Fonte: Unesp, Sistema de Base de Dados da Graduação - SISGrad: 2014-2018.

O Gráfico 4 evidencia que o movimento do índice anual de desistência dos cursos é semelhante nas três categorias de ingresso: SU, EP e PPI (com um pico em 2016, diminuindo em 2017 e 2018). Entre as três categorias, o índice, no período observado, é sempre maior entre os PPI, intermediário para os ingressantes da escola pública, mas não enquadrados entre os PPI, e inferior para os ingressantes do SU. Embora pareça ser uma tendência clara, quando se consideram os percentuais de desistência, deve ser considerado o que foi mostrado anteriormente com base nos Gráficos 2 e 3, ou seja, a diferença significativa de dimensão entre 
as três categorias, e o fato de o tamanho do universo ainda não ter se estabilizado para cada uma delas, uma vez que o sistema de inclusão da Unesp ainda estava em implantação, no recorte temporal efetuado. Desta maneira, para minimizar os efeitos da variação do número de alunos nos diferentes cursos, decidiu-se realizar um teste pareado, a fim de verificar a efetividade dos resultados apresentados no Gráfico 4, avaliando se, de fato, as categorias EP e PPI apresentam maior risco de evasão no contexto dos cursos.

\section{Gráfico 4 - Índices anuais de evasão dos cursos da Unesp, por categoria de ingresso (SU, EP, PPI)}

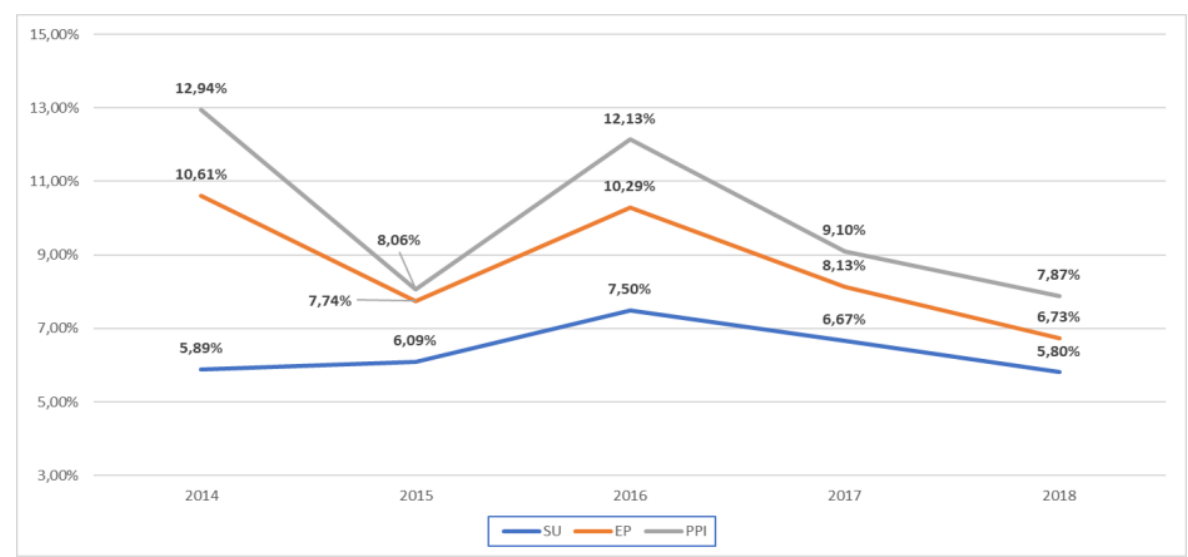

Fonte: Unesp, Sistema de Base de Dados da Graduação - SISGrad: 2014-2018.

Desse modo, pode-se verificar se existem ou não diferenças estatisticamente significativas nos percentuais de evasão entre as diferentes categorias de ingresso. O Gráfico 5 e a Tabela 1 expressam os valores das taxas para diferentes cursos. As barras do referido gráfico representam os maiores e menores valores. As barras inferiores referem-se ao primeiro e ao terceiro quartil, a linha indica o valor da média. As barras superiores marcam os casos em que houve diferença estatística.

Com base no teste pareado, foram obtidos os resultados apresentados no Gráfico 5 e na Tabela 1, referentes ao conjunto de cursos da Unesp. Esses achados revelam que, apesar de a dispersão dos dados ser maior na categoria de PPI, e de haver alguma diferença de comportamento entre as categorias de ingresso nos dois primeiros anos observou-se também uma diferença entre os grupos PPI e SU e entre PPI e EP nos dois últimos anos. O tamanho do efeito é de aproximadamente 2 pontos percentuais. Não se observa nenhuma diferença entre EP e SU. Isso evidencia que os PPIs são mais frágeis que os oriundos de escolas públicas. 
Gráfico 5 - Taxa de Evasão por Curso, por categoria (SU, EP, PPI)
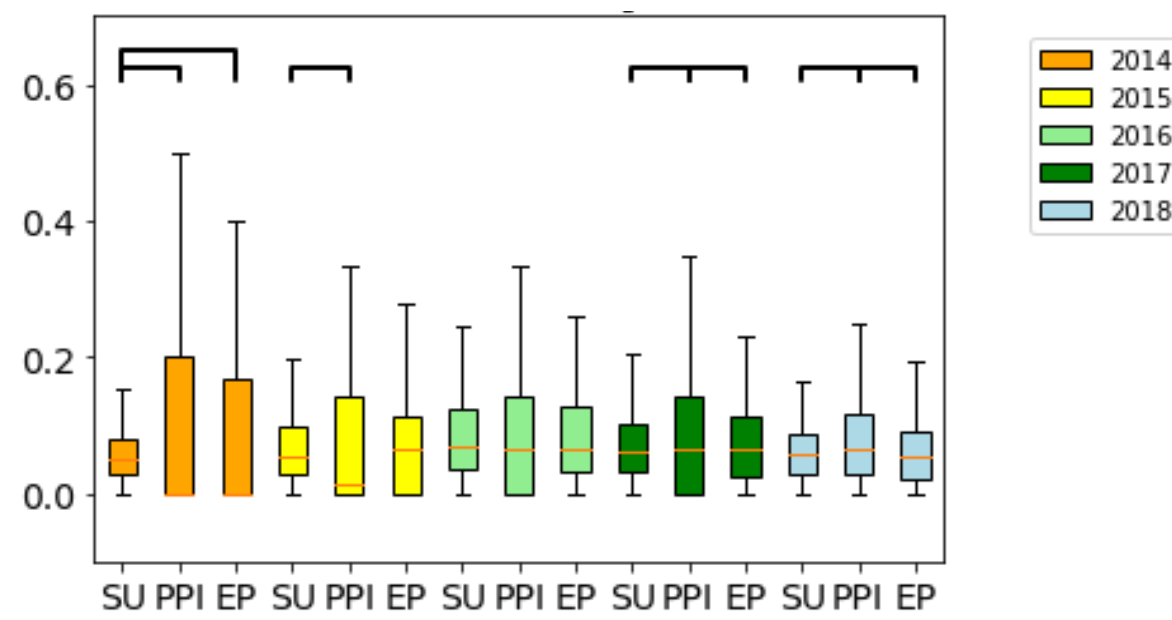

Fonte: Unesp, Sistema de Base de Dados da Graduação - SISGrad: 2014-2018.

Tabela 1 - Taxa de Evasão por Curso, por categoria (SU, EP, PPI)

\begin{tabular}{|c|c|c|c|c|c|c|c|c|c|}
\hline \multirow{2}{*}{ Ano } & \multicolumn{2}{|c|}{ SU } & \multirow{2}{*}{$\begin{array}{c}\text { PPI } \\
\mu\end{array}$} & \multicolumn{3}{|c|}{ EP } & \multirow{2}{*}{$\frac{\text { SUx PPI }}{\text { p-value }}$} & \multirow{2}{*}{$\frac{\text { SUx EP }}{\text { p-value }}$} & \multirow{2}{*}{$\frac{\text { EPPx PPI }}{\text { p-value }}$} \\
\hline & $\mu$ & $\sigma$ & & $\sigma$ & $\mu$ & $\sigma$ & & & \\
\hline 2014 & 0.068 & 0.0637 & 0.115 & 0.192 & 0.0893 & 0.0012 & 0.001 & 0.016 & 0.139 \\
\hline 2015 & 0.0705 & 0.063 & 0.0942 & 0.136 & 0.0752 & 0.0848 & 0.011 & 0.386 & 0.0642 \\
\hline 2016 & 0.091 & 0.0768 & 0.0941 & 0.114 & 0.0898 & 0.0824 & 0.698 & 0.833 & 0.531 \\
\hline 2017 & 0.0786 & 0.0677 & 0.0921 & 0.093 & 0.08 & 0.0759 & 0.018 & 0.833 & 0.0271 \\
\hline 2018 & 0.0666 & 0.0535 & 0.0799 & 0.072 & 0.0688 & 0.0698 & 0.009 & 0.588 & 0.0435 \\
\hline
\end{tabular}

Fonte: Unesp, Sistema de Base de Dados da Graduação - SISGrad: 2014-2018.

Quando se consideram as grandes áreas (Exatas, Humanas e Biológicas), verificam-se pequenas alterações de comportamento nos grupos de ingresso, quanto à evasão, que podem ser observadas a seguir.

Os dados do Gráfico 6 e da Tabela 2 permitem notar que, de maneira geral, no período observado, o grupo de ingresso dos PPI apresenta um risco maior de evasão, quando comparado aos alunos oriundos da escola pública (EP) e que ingressaram pelo SU. Mas este comportamento não se confirma nas demais grandes áreas. 
Gráfico 6 - Taxa de Evasão na área de Ciências Exatas, por categoria (SU, EP, PPI)

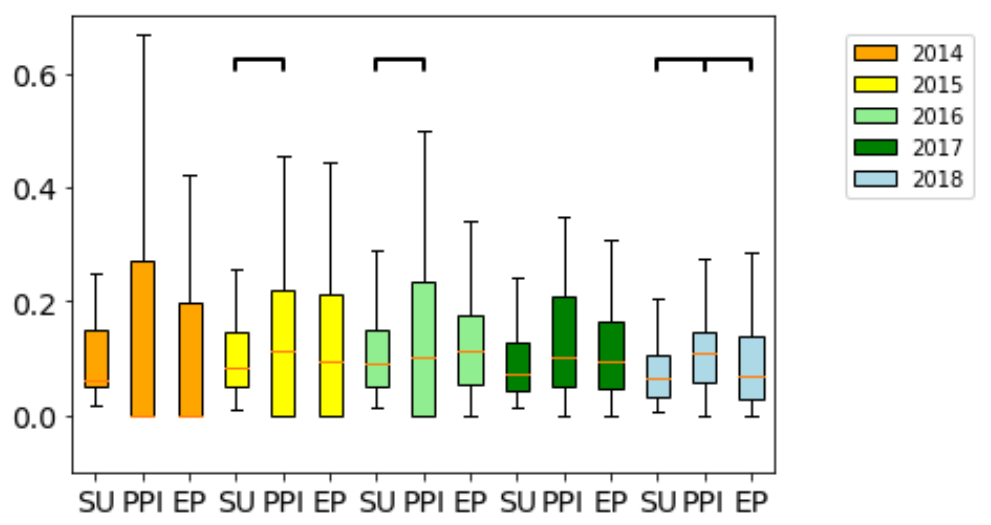

Fonte: Unesp, Sistema de Base de Dados da Graduação - SISGrad: 2014-2018.

Tabela 2 - Taxa de Evasão na área de Ciências Exatas, por categoria (SU, EP, PPI)

\begin{tabular}{c|c|c|c|c|c|c|c|c|c|}
\hline \multirow{2}{*}{ Ano } & \multicolumn{2}{c}{ SU } & \multicolumn{2}{c}{ PPI } & \multicolumn{2}{c}{ EP } & SU x PPI & SU x EP & EPP x PPI \\
\cline { 2 - 10 } & $\boldsymbol{\mu}$ & $\boldsymbol{\sigma}$ & $\boldsymbol{\mu}$ & $\boldsymbol{\sigma}$ & $\boldsymbol{\mu}$ & $\boldsymbol{\sigma}$ & $\mathbf{p}$-value & p-value & p-value \\
\hline 2014 & 0.101 & 0.0846 & 0.165 & 0.248 & 0.105 & 0.131 & 0.0678 & 0.815 & 0.121 \\
\hline 2015 & 0.106 & 0.08 & 0.155 & 0.171 & 0.116 & 0.107 & 0.0174 & 0.417 & 0.0939 \\
\hline 2016 & 0.108 & 0.075 & 0.146 & 0.157 & 0.126 & 0.0946 & 0.0447 & 0.0963 & 0.198 \\
\hline 2017 & 0.104 & 0.0816 & 0.126 & 0.108 & 0.114 & 0.0952 & 0.0772 & 0.303 & 0.308 \\
\hline 2018 & 0.0794 & 0.0636 & 0.116 & 0.088 & 0.0913 & 0.0823 & 0.0006 & 0.101 & 0.0291 \\
\hline
\end{tabular}

Fonte: Unesp, Sistema de Base de Dados da Graduação - SISGrad: 2014-2018.

Como mostram o Gráfico 7 e a Tabela 3, na grande área de Ciências Biológicas, em 2017, o grupo de ingressantes em SU apresenta menor risco de se evadir, quando comparado com PPI e EP. Apenas em 2018 é possível verificar que os PPI têm maior risco de evadir do que os EP.

Gráfico 7 -Taxa de Evasão na área de Biológicas, por categoria (SU, EP, PPI)

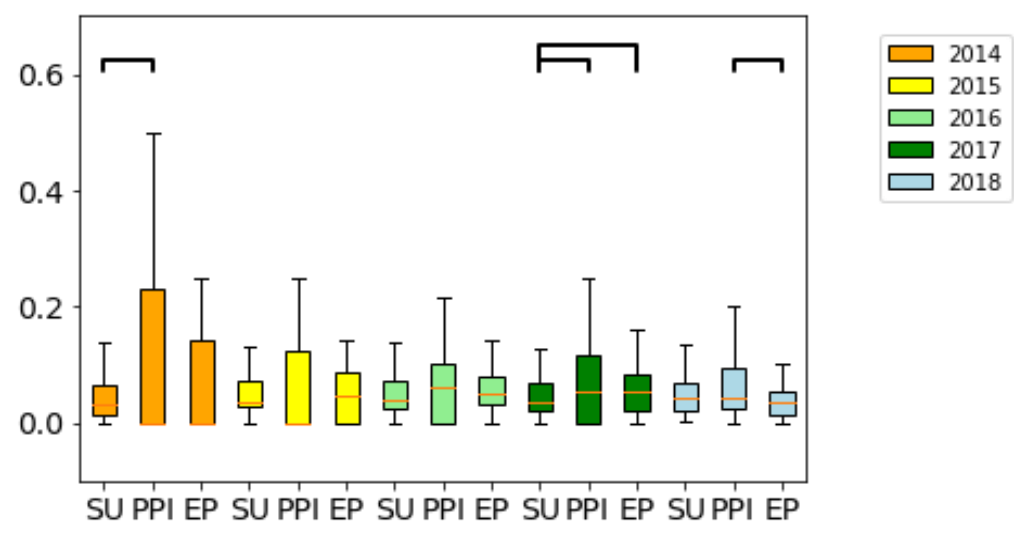


Fonte: Unesp, Sistema de Base de Dados da Graduação - SISGrad: 2014-2018.

Tabela 3 - Taxa de Evasão na área de Biológicas, por categoria (SU, EP, PPI)

\begin{tabular}{|c|c|c|c|c|c|c|c|c|c|}
\hline \multirow{2}{*}{ Ano } & \multicolumn{2}{|c|}{ SU } & \multicolumn{2}{|c|}{ PPI } & \multicolumn{2}{|c|}{ EP } & \multirow{2}{*}{$\frac{\text { SU x PPI }}{\text { p-value }}$} & \multirow{2}{*}{$\begin{array}{c}\text { SU x EP } \\
\text { p-value }\end{array}$} & \multirow{2}{*}{$\frac{\text { EPP x PPI }}{\text { p-value }}$} \\
\hline & $\mu$ & $\sigma$ & $\mu$ & $\sigma$ & $\mu$ & $\sigma$ & & & \\
\hline 2014 & 0.05 & 0.0526 & 0.101 & 0.157 & 0.0799 & 0.11 & 0.0237 & 0.0599 & 0.393 \\
\hline 2015 & 0.051 & 0.0365 & 0.0673 & 0.09 & 0.0508 & 0.0563 & 0.238 & 0.981 & 0.281 \\
\hline 2016 & 0.052 & 0.0386 & 0.0647 & 0.068 & 0.0602 & 0.0545 & 0.182 & 0.316 & 0.635 \\
\hline 2017 & 0.0442 & 0.0321 & 0.0693 & 0.067 & 0.0573 & 0.0487 & 0.0088 & 0.0288 & 0.142 \\
\hline 2018 & 0.0489 & 0.0324 & 0.0617 & 0.056 & 0.0413 & 0.0434 & 0.114 & 0.233 & 0.0092 \\
\hline
\end{tabular}

Fonte: Unesp, Sistema de Base de Dados da Graduação - SISGrad: 2014-2018.

Por sua vez, o Gráfico 8 e a Tabela 4 informam que, na grande área de Ciências Humanas, em 2018, o risco de evasão é equivalente nas três categorias. Em 2017, os SU têm maior risco de evadir, quando comparados com EP. Também em 2016 o risco de evasão em SU era maior, quando comparado com PPI e EP.

Gráfico 8 - Taxa de Evasão na área de Humanas, por categoria (SU, EP, PPI)

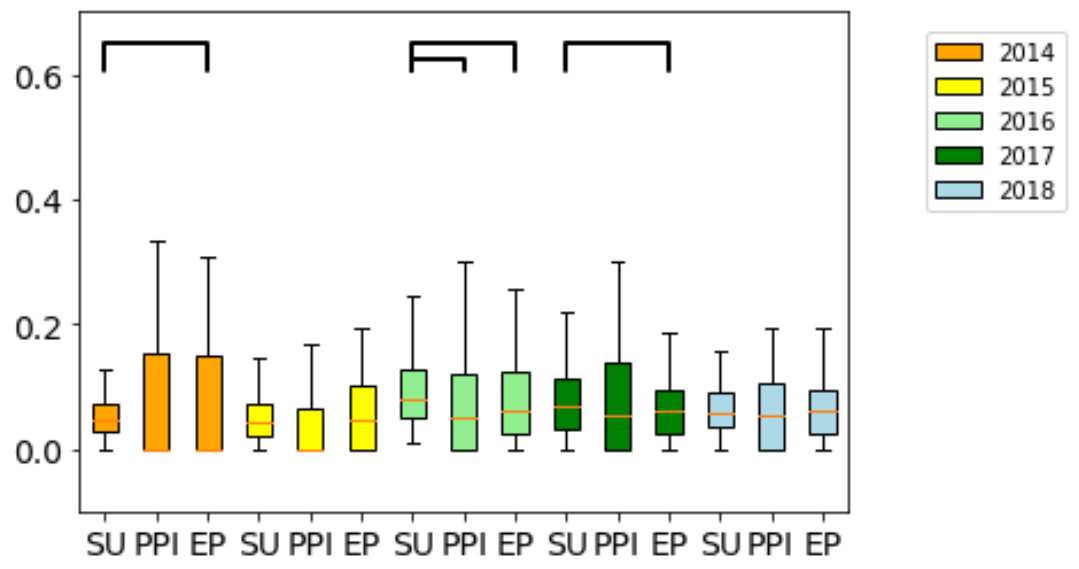

Fonte: Unesp, Sistema de Base de Dados da Graduação - SISGrad: 2014-2018. 
Tabela 4 - Taxa de Evasão na área de Humanas, por categoria (SU, EP, PPI)

\begin{tabular}{|c|c|c|c|c|c|c|c|c|c|}
\hline \multirow{2}{*}{ Ano } & \multicolumn{2}{|c|}{ SU } & \multicolumn{2}{|c|}{ PPI } & \multicolumn{2}{|c|}{ EP } & \multirow{2}{*}{$\frac{\text { SU x PPI }}{\text { p-value }}$} & \multirow{2}{*}{$\frac{\text { SU } x \text { EP }}{\text { p-value }}$} & \multirow{2}{*}{$\frac{\text { EPP x PPI }}{p \text {-value }}$} \\
\hline & $\mu$ & $\sigma$ & $\mu$ & $\sigma$ & $\mu$ & $\sigma$ & & & \\
\hline 2014 & 0.0527 & 0.0337 & 0.0844 & 0.153 & 0.0826 & 0.118 & 0.0854 & 0.0418 & 0.979 \\
\hline 2015 & 0.0549 & 0.0475 & 0.0629 & 0.113 & 0.0583 & 0.068 & 0.541 & 0.634 & 0.741 \\
\hline 2016 & 0.103 & 0.0881 & 0.0714 & 0.079 & 0.0796 & 0.077 & 0.0028 & 0.0081 & 0.408 \\
\hline 2017 & 0.0803 & 0.0633 & 0.0797 & 0.088 & 0.0674 & 0.0633 & 0.942 & 0.0473 & 0.132 \\
\hline 2018 & 0.0676 & 0.053 & 0.0621 & 0.055 & 0.0684 & 0.0669 & 0.433 & 0.917 & 0.453 \\
\hline
\end{tabular}

Fonte: Unesp, Sistema de Base de Dados da Graduação - SISGrad: 2014-2018.

A modalidade do curso escolhida pelo aluno - Bacharelado, Licenciatura ou Bacharelado/Licenciatura - também interfere no risco de evasão, como demonstram os Gráficos 9, 10 e 11. Nos cursos em que ambas as modalidades - Bacharelado e Licenciatura (Gráfico 11) - são oferecidas concomitantemente e nas Licenciaturas (Gráfico 10), o risco de evasão é equivalente nas três categorias. Ao passo que nos Bacharelados (Gráfico 9), os PPI apresentam maior risco de evasão, quando comparados com SU, em 2017 e 2018. Em 2018, o risco de evasão é maior nos PPI também em comparação com EP.

Gráfico 9 - Taxa de Evasão nos cursos de Bacharelado, por categoria (SU, EP, PPI)

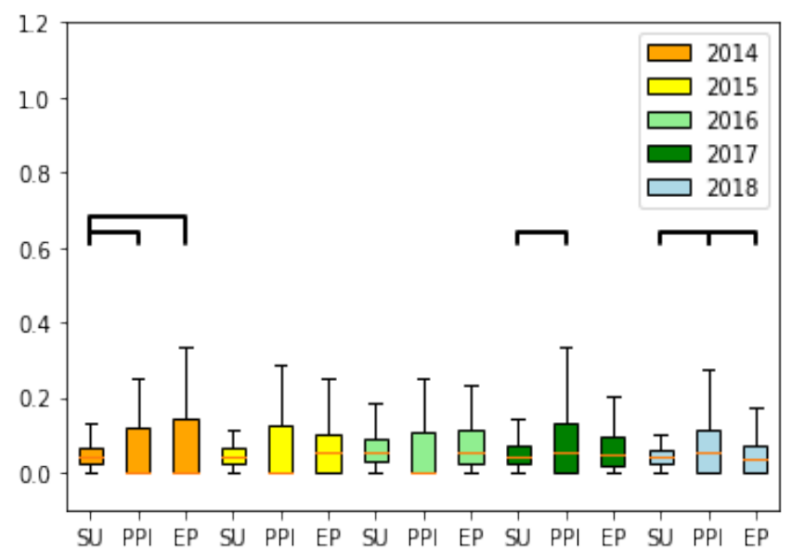

Fonte: Unesp, Sistema de Base de Dados da Graduação - SISGrad: 2014-2018. 
Gráfico 10 - Taxa de Evasão nos cursos de Licenciatura, por categoria (SU, EP, PPI)

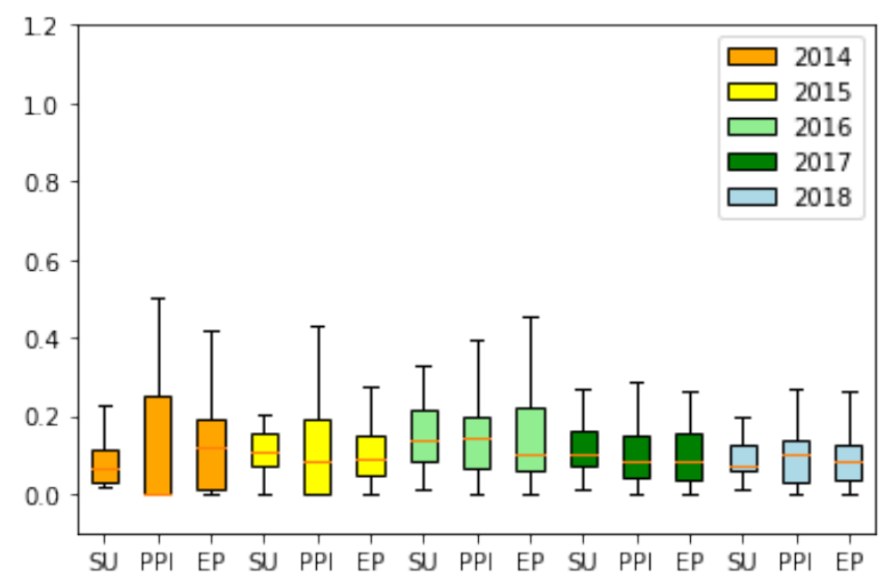

Fonte: Unesp, Sistema de Base de Dados da Graduação - SISGrad : 2014-2018

Gráfico 11 - Taxa de Evasão nos cursos de Bacharelado e Licenciatura, por categoria (SU, EP, PPI)

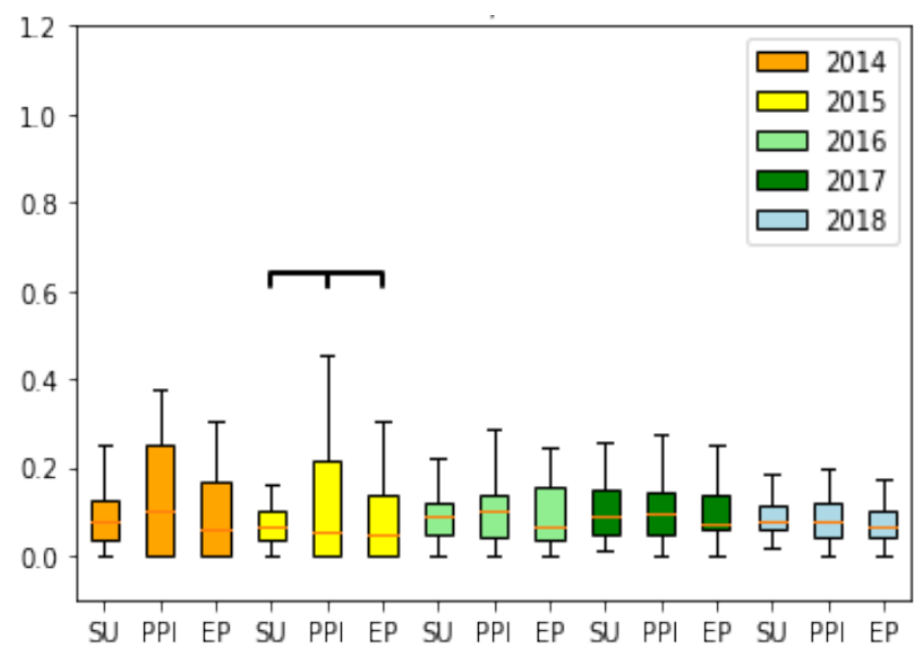

Fonte: Unesp, Sistema de Base de Dados da Graduação - SISGrad: 2014-2018.

Em termos de período de oferecimento (diurno/integral; matutino; vespertino; vespertino/noturno; noturno), não foram observadas diferenças significativas, que pudessem indicar um período específico que pudesse favorecer ou desfavorecer a desistência dos cursos.

\section{Discussão e conclusões}

Chegar ao ensino superior não é uma realidade fácil na sociedade contemporânea e explicar a evasão envolve questões complexas que precisam ser reconhecidas, na busca de resoluções. Tinto (2006) salienta que analisar como o grau, a capacidade e o status social de um indivíduo se relacionam com a probabilidade da evasão do ensino superior não significa saber como esses atributos afetam o processo de desistência. Enquanto o primeiro exige pouco mais do que uma simples comparação das taxas de abandono entre indivíduos com diferentes 
habilidades e características de status social, o segundo exige o desenvolvimento de um modelo longitudinal teórico que vincule várias características individuais e institucionais ao processo de evasão.

Os índices anuais de evasão da Unesp, 6,7\% (2016) e 5,1\% (2018) se mostraram abaixo da média nacional para as IES públicas em cursos presenciais, respectivamente 27,2 \% (2016) e 18,5\% (2018), segundo Censo da Educação Superior 2018 (INEP 2019). A política de reserva de vagas e cotas, no recorte de tempo citado, mostra uma relação com a redução das taxas de evasão na Unesp. De acordo com Silva Filho e Lobo (2012), as causas de evasão relacionadas a questões socioeconômicas podem elevar discretamente as taxas anuais. $\mathrm{Na}$ Unesp, quando se consideram apenas os percentuais de alunos que desistem dos cursos, comparativamente entre as três categorias de ingresso como mostrou o Gráfico 4, a evasão nas categorias de EP e PPI é ligeiramente mais alta do que em SU. Essas diferenças percentuais advêm da observação de universos de dados muito assimétricos, em que nas análises baseadas em testes pareados, apontaram que, no plano geral da universidade, o comportamento da evasão nas três categorias de ingresso é semelhante.

Acredita-se que este fenômeno tem ocorrido na Unesp em virtude de ações efetivas de permanência estudantil implementadas pela gestão. De acordo com Tinto (2006), políticas de permanência estudantil bem-sucedidas devem estar atreladas aos aspectos sociais da vida acadêmica. A amplitude e a variedade de tais políticas na Unesp, com programas desenhados com essa abordagem, ajudam a explicar essa redução, uma vez que contempla o processo de inclusão das diferenças e na aceitação da diversidade, como o projeto de extensão "Educando para Diversidade", restaurantes universitários, moradia estudantil, bolsas para alunos em situação de fragilidade socioeconômica e criação de equipes multidisciplinares de apoio aos estudantes.

Esta observação é importante para tomada de decisões relativas a possíveis causas de evasão na Unesp, a saber: causas associadas a dificuldades prévias de aprendizado em disciplinas básicas, por exemplo: cálculo. A qualidade do ensino médio e dos últimos anos da educação básica, bem como a aproximação da universidade com estes níveis de ensino, são variáveis importantes quando se analisa a evasão nos alunos ingressantes (VITELLI; FRITSCH, 2016).

O despreparo dos alunos ingressantes no ensino superior, oriundos de ensino médio deficiente, pode determinar um fenômeno denominado "fragilidade do estudante ingressante", caracterizado pela falta de metodologia para estudo, autonomia frágil e não reconhecimento do processo ensino-aprendizagem (VITELLI; FRITSCH, 2016). Assim, o acompanhamento 
criterioso por parte da instituição, com ações acadêmicas e psicossociais, é determinante para a diminuição dos índices de evasão.

Quando se analisa o resultado da evasão nos cursos da grande área de Ciências Humanas, cujo índice maior está entre o SU, reporta-se às conclusões de Cunha e Morosini (2013), que agruparam as causas de evasão em três grandes grupos: a) causas pessoais; b) institucionais e c) gerais. As causas pessoais estão associadas a dificuldade da integração ao mercado de trabalho, baixa remuneração profissional, escolha prematura da profissão e podem explicar o abandono entre os egressos do SU na área de Ciências Humanas. Muitas vezes, estes cursos podem estar associados à melhoria do currículo para o exercício de outras profissões. Estas mesmas explicações podem ser inferidas nos resultados obtidos quando foram analisadas as diferentes modalidades dos cursos: nos cursos de Bacharelado, o maior índice de evasão recai no grupo dos PPI e nos cursos de formação de professores (Licenciaturas e Bacharelados/Licenciaturas), a evasão é semelhante nas três categorias de ingresso.

Finalmente, estes resultados explicitam e reforçam a importância do SRVEBP na inclusão e equidade de oportunidades ao ingresso no ensino superior, demonstrando que o índice de evasão se mantém menor que a média nacional. Entre as causas mais usuais para a evasão em nível mundial estão problemas financeiros, formação inadequada e dificuldades de adaptação ao ambiente universitário. Sob esses aspectos a retenção dos cotistas representa considerável desafio, pois tipicamente eles acabam por se enquadrar em algum desses perfis. Nos Gráficos 2 e 3 fica evidente que a política de cotas ocorreu concomitantemente à redução das taxas de evasão e aliada a medidas e permanência estudantil supramencionadas.

Os dados coletados a respeito dos números gerais sobre evasão mostram que a política de cotas, muito antes de causar uma diminuição na retenção, ocorreu em um momento em que houve diminuição da evasão. Colocando em dúvida algumas das análises apresentadas por Schwartzman (2008), ainda que seja necessária uma janela temporal muito mais extensa para se tirar conclusões definitivas, cabem algumas considerações. Um aspecto relevante desconsiderado nessa análise é o quadro de rápidas e disruptivas transformações no ensino superior. Nesse novo quadro, os cotistas tendem a valorizar mais fortemente a oportunidade de ascensão social representada pelo acesso à universidade, seja porque isso os destaca em seu meio social e familiar, seja porque percebem as vantagens que o networking do meio acadêmico possa ter em sua futura vida profissional. A inclusão via cotas tem, além de qualquer sentido objetivo, traz um efeito simbólico que não pode ser desprezado, o acesso ou mesmo a possibilidade de acesso a universidades de excelência que impacta de forma tangível as 
perspectivas de setores menos favorecidos e na imagem externa que a universidade projeta na sociedade.

Os resultados obtidos com base nos testes pareados indicaram, também, algumas pequenas diferenças de comportamento quanto ao risco de evasão dos cursos, quando se consideram as grandes áreas nas quais esses cursos estão inseridos. Um importante fato a ser observado é que o maior risco de evasão se inverte, quando se comparam, por um lado, as áreas de Ciências Exatas e Biológicas, com os cursos das áreas de Ciências Humanas. Ao passo que nos cursos da área de Exatas o maior risco de evasão está no grupo dos PPI, quando comparados aos ingressantes em SU e EP, e nas Ciências Biológicas se verifica maior risco de evasão ligeiramente mais elevado no grupo dos PPI, nas Ciências Humanas, o maior risco de evasão recai no grupo dos ingressantes pelo SU.

Entre as modalidades (Bacharelado, Licenciatura e Bacharelado/Licenciatura), também se verifica um comportamento assimétrico quando se consideram, por um lado, os cursos de Bacharelado, em que o maior risco de evasão recai no grupo dos PPI e, por outro, os cursos de formação de professores (Licenciaturas e Bacharelados/Licenciaturas), que revelam um comportamento semelhante da evasão nas três categorias de ingresso.

De maneira geral, o que a análise dos dados realizada por esta pesquisa mostra é a importância social da adoção do SRVEBP no vestibular da Unesp, uma vez que, na média geral da universidade, não há diferenças significativas no risco de evasão apresentado nas três categorias de ingresso (SU, EP, PPI). Este fato revela que o projeto de inclusão adotado progressivamente pela universidade não trouxe riscos à qualidade de seus cursos. Pelo contrário, em alguns casos específicos (como na área de Ciências Humanas), as categorias relacionadas aos estudantes que cursaram o ensino médio integralmente em escolas públicas (EP e PPI) estão relacionadas a um menor risco de evasão - o que leva, nestes casos específicos, à conclusão de que a adoção do SRVEBP promove um bom preenchimento, em termos qualitativos, das vagas oferecidas, no que diz respeito à possibilidade de finalização do curso pelo ingressante e à sua possibilidade de ascensão social, o que projeta um bom retorno ao investimento público na formação dos futuros profissionais. É necessário ressaltar, por fim, que a reserva de vagas se estende a todas as turmas de todos os cursos e de todos os períodos, o que amplia a importância social do projeto de inclusão aos cursos de todas as áreas, em todos os câmpus da Unesp. 


\section{Referências}

ARULAMPALAM, Wiji; NAYLOR, Robin A.; SMITH, Jeremy. A hazard model of the probability of medical school dropout in the United Kingdom. IZA Discussion Paper, Bonn, Alemanha, n. 333, 2001. Disponível em: http://ftp.iza.org/dp333.pdf. Acesso em: 18 fev. 2020.

BEZERRA, Teresa Olinda Caminha. A política pública de cotas em universidades, desempenho acadêmico e inclusão social. Sustainable Business International Journal, Niterói, n. 9, p. 1-22, 2011.

BLANCHFIELD, William C. College dropout identification: an economic analysis. Journal of Human Resources, Madison, v. 7, n. 4, p. 540-544, 1972.

BOOTH, Alison L.; SATCHELL, Stephen E. The hazards of doing a PhD: an analysis of completion and withdrawal rates of British PhD students in the 1980s. Journal of the Royal Statistical Society. Series A (Statistics in Society), Hoboken, v. 158, n. 2, p. 297-318, 1995.

BRADLEY, Steve; LENTON, Pam. Dropping out of post-compulsory education in the UK: an analysis of determinants and outcomes. Lancaster, UK: Department of Economics, Management School, Lancaster University, 2005. (Working Paper).

COMISSÃO ESPECIAL DE ESTUDOS SOBRE A EVASÃO NAS UNIVERSIDADES PÚBLICAS BRASILEIRAS. Diplomação, retenção e evasão nos cursos de graduação em instituições de ensino superior públicas. Brasília: ANDIFES/ABRUEM/SESu/MEC, 1996. Disponível em: http://goo.gl/bE3y8t. Acesso em: 9 maio 2018.

CUNHA, Emmanuel Ribeiro; MOROSINI, Marília Costa. Evasão na educação superior: uma temática em discussão. Revista Cocar, Belém, v. 7, n. 14, p. 82-89, ago./dez. 2013.

DESJARDINS, Stephen et al. An event history model of student departure. Economics of Education Review, Amsterdam, v. 18, n. 3, p. 375-390, 1999.

ECKSTEIN, Zvi; WOLPIN, Kenneth I. Why youths drop out of high school: the impact of preferences, opportunities, and abilities. Econometrica, Chicago, v. 67, n. 6, p. 1295-1339, 1999.

EHRENBERG, Ronald; MAVROS, Panagiotis G. Do Doctoral students' financial support patterns affect their times-to-degree and completion probability? Journal of Human Resources, Madison, v. 30, n. 3, p. 581-609, 1995.

FISHER, Ronald A. On the interpretation of $\chi^{2}$ from contingency tables, and the calculation of P. Journal of the Royal Statistical Society, Hoboken, v. 85, n. 1, p. 87-94, 1922.

INSTITUTO NACIONAL DE ESTUDOS E PESQUISAS EDUCACIONAIS ANÍSIO TEIXEIRA (Inep). Censo da Educação Superior 2018: notas estatísticas. Brasília: INEP, 2019. 
MALLETE, Bruce I.; CABRERA, Alberto F. Determinants of withdrawal behavior: an exploratory study. Research in Higher Education, New York, v. 32, n. 2, p. 179-194, 1991.

MASSINI-CAGLIARI, Gladis et al. O objetivo faz o método: perspectivas para o cálculo do índice anual de evasão na Unesp. In: MANCHOPE, Elenita Conegero Pastor et al. (org.).

Interiorização do ensino superior: Protagonismo das universidades estaduais e municipais no desenvolvimento regional. Cascavel: Edunioeste, 2018. p. 127-140.

PANTAGES, Timothy J.; CREEDON, Carol F. Studies of college attrition: 1950-1975. Review of Educational Research, Philadelphia, v. 48, n. 1, p. 49-101, 1978.

SCHWARTZMAN, Simon. A questão da inclusão social na universidade brasileira. In: PEIXOTO, Maria do Carmo de Lacerda; ARANHA, Antônia Vitória Soares. (org.). Universidade pública e inclusão social: experiência e imaginação. Belo Horizonte: Editora UFMG, 2008. p. 23-43.

SIEGFRIED, John J.; STOCK, Wendy A. So you want to earn a Ph.D. in economics? How long do you think it will take? Journal of Human Resources, Madison, v. 36, n. 2, p. 364$378,2001$.

SILVA FILHO, Roberto Leal; LOBO, Maria Beatriz de Carvalho Melo. Esclarecimentos metodológicos sobre os cálculos de evasão. Mogi das Cruzes: Instituto Lobo, 2012.

Disponível em: http://www.institutolobo.org.br/imagens/pdf/artigos/art_078.pdf. Acesso em: 9 maio 2018.

SIMPSON, Carl; BAKER, Kathryn; MELLINGER, Glen. Conventional failures and unconventional dropouts: comparing different types of university withdrawals. Sociology of Education, Philadelphia, v. 53, n. 4, p. 203-214, 1980.

STUDENT. The Probable Error of a Mean. Biometrika, London, v. 6, n. 1, p. 1-25, mar. 1987.

TINTO, Vincent. Leaving College: rethinking the causes and cures of student attrition. Chicago, IL: University of Chicago Press, 1987.

TINTO, Vincent. Research and pratice of student retention, what next? J. CoLLege Student Retention, Philadelphia, v. 8, p. 1-19, 2006.

VASCONCELOS, Mário Sérgio; GALHARDO, Eduardo. O Programa de inclusão na UNESP: Valores, contradições e ações afirmativas. Revista Ibero-Americana de Estudos em Educação, Araraquara, v. 11, n. esp. 1, p. 285-306, 2016.

VITELLI, Ricardo Ferreira; FRITSCH, Rosangela. Evasão escolar na educação superior: de que indicador estamos falando? Estudos em Avaliação Educacional, São Paulo, v. 27, n. 66, p. 908-937, 2016. Disponível em: http://conference.uniso.br/index.php/epic/2018/paper/view/1287/150. Acesso em: 5 fev. 20 